\title{
Evaluation of Changes in T-wave Alternans Induced by 60-days of Immobilization by Head-Down Bed-Rest
}

\author{
Alba Martín-Yebra ${ }^{1,2}$, Violeta Monasterio ${ }^{3}$, Pablo Laguna ${ }^{2,4}$, Juan Pablo Martínez ${ }^{2,4}$, Enrico G \\ Caiani $^{1}$ \\ ${ }^{1}$ Dipartimento di Elettronica, Informazione e Bioingegneria, Politecnico di Milano, Milan, Italy \\ ${ }^{2}$ BSICoS Group, Aragón Institute of Engineering Research, IIS Aragón, Universidad de Zaragoza, \\ Zaragoza, Spain \\ ${ }^{3}$ Universidad San Jorge, Villanueva de Gállego, Zaragoza, Spain \\ ${ }^{4}$ CIBER en Bioingeniería, Biomateriales y Nanomedicina (CIBER-BBN), Spain
}

\begin{abstract}
Prolonged exposure to weightlessness is known to induce cardiovascular deconditioning. The aim of this work was to assess changes in T-wave alternans (TWA) induced by 60 days exposure to simulated microgravity using the $-6^{\circ}$ head-down bed-rest model (HDBR) and the potential effectiveness of a jump training countermeasure. We hypothesized that TWA could be able to reflect these changes, if they exist. Twenty-four healthy men were recruited at the German Aerospace Center (12 in control group, 12 with applied countermeasure), from which ECG signals were acquired before (PRE), at day 21 of HDBR (HDT21), before the end of HDBR (HDT57), and the day after its conclusion (POST). The index of average alternans (IAA), quantifying the average TWA amplitude, was computed using a fully automated algorithm based on periodic component analysis and the Laplacian likelihood ratio method. A significant increase in HR was found at HDT57 and POST compared to PRE. The IAA showed an increasing trend at POST (median (25th; 75th percentile): $0.467(0.381 ; 0.574) \mu V)$ compared to PRE $(0.337(0.204 ; 0.437) \mu V, p=0.18)$ only in the control group, although no significant differences along HDBR were found. In conclusion, long-term exposure to simulated microgravity did not induce significant alterations in electrical instability measured in terms of nocturnal TWA.
\end{abstract}

\section{Introduction}

Prolonged weightlessness condition leads to cardiovascular deconditioning, inducing changes in autonomic control of cardiovascular system [1]. This arises several alterations in the organism, with potential impact also in cardiac electrical activity. Thus, cardiac rhythm disturbances may be originated if cardiac repolarization is adversely influen- ced. In particular, anecdotal data of cardiac arrhythmias and conduction disorders during spaceflight [2] and higher incidence of ventricular premature contractions [3], together with reports that long-duration spaceflight, but not short-duration ones, led to the prolongation of the QTc interval in crewmembers $[4,5]$, suggested that weightlessness can potentially increase arrhythmia susceptibility.

Since research under real conditions of spaceflights is limited, the head-down $\left(-6^{\circ}\right)$ bed-rest (HDBR) model has been proved to be a reliable ground-based simulation ana$\log$ [6]. This manoeuvre represents a model of chronic circulatory unloading simulating sustained exposure to microgravity and offers a unique opportunity to study the effects of prolonged spaceflight on the cardiovascular system, as well as to test potential countermeasures to prevent its deconditioning.

Recently, spatial and temporal heterogeneity of ventricular repolarization induced by short (5-days) [7] and long duration (90-days) [8] HDBR were investigated. Both studies confirmed that HDBR induced a reversible increase in ECG repolarization heterogeneity by an increase in QRS$\mathrm{T}$ angle and a decrease in the spatial ventricular gradient, thus supporting the hypothesis of increased ventricular arrhythmic risk.

Based on all previous observations, we hypothesized that long-duration HDBR induces reversible increase in ventricular repolarization heterogeneity that could be manifested by an increase on T-wave alternans (TWA), a consistent beat-to-beat alternation in the amplitude or morphology of the ST segment and/or the T wave, indicating an increased ventricular arrhythmic risk [9]. Accordingly, our aim in this study was to assess changes on TWA induced by 60 days exposure to simulated microgravity. 


\section{Study population}

One long-duration (60 days) HDBR campaign was performed at the German Aerospace Center (DLR) in the context of the European Space Agency HDBR strategy, including 24 subjects with age range 21-42 years (30 \pm 6$)$, weight $77 \pm 7 \mathrm{~kg}$ and height $181 \pm 6 \mathrm{~cm}$. Subjects were randomly allocated in control (CNTRL, $n=12)$ or in the intervention group (JUMP, $n=12$ ), where applied intervention to be tested in this case consisted in reactive jumps in a sledge jump system in a supine position, as a countermeasure for muscle and bone loss. A typical training session consisted of $4 \times 10$ countermovement jumps and 2x10 hops. The JUMP group had to complete 56 sessions per week (approximately 3 min per session). One subject from control group dropped out for medical reasons not related to the study. All volunteers provided written informed consent to participate in the study approved by the respective Ethical Committee for Human Research at the hosting institution.

The protocol included some days of acclimation to the bed rest facility (referred as PRE period), for baseline data collection. During the HDBR period, all subjects stayed monitored, in strict $6^{\circ}$ negative head-down tilt bed-rest, $24 \mathrm{~h}$ a day for 60 days. In this period (referred as HDTX, where $\mathrm{X}$ is the day number of HDBR), subjects were awakened at 6:30 AM and prompted to start sleeping at 11:00 PM each day, with no napping allowed during the day. After 60 days of HDBR, subjects remained in the facility for some additional days (referred as POST). During the PRE and POST periods, lying in bed during the day was not allowed. A more detailed description of the experimental protocol can be found in [10].Twenty-four-hour Holter ECG recordings (H12+, Mortara Instrument Inc., Milwaukee, WI, 12 leads, $1000 \mathrm{~Hz}$ sampling frequency) were acquired at PRE, at days HDT21 and HDT57 during HDBR and at POST.

\section{Methods}

\section{1. $\quad$ Preprocessing}

Preprocessing of ECG recordings included QRS detection using a wavelet-based delineator [11] and linear filtering for baseline wander removal. Then ECG was lowpass filtered and down-sampled to $125 \mathrm{~Hz}$ to reduce noise and the computational cost of TWA analysis. Finally, the ST-T complex was windowed at each beat, by defining a fixed interval of $350 \mathrm{~ms}$ (reduced to 44 samples after decimation) after the end of the QRS complex.

\subsection{TWA analysis}

TWA analysis was performed by analyzing Holter ECGs portions corresponding to the night period (23.00-06.00) in order to avoid potential confounding effects (subjects were subjected to other experiments). Automatic TWA estimation for each recording was performed in three steps: (1) selection of suitable ECG segments, (2) estimation of the TWA amplitude in each segment and (3) computation of the index of average alternans $(I A A)$ activity that characterizes each recording.

ECG signals were processed in segments of $L=128$ consecutive beats (with $50 \%$ overlap). In order to exclude possible transients existing in the signal, a stability criterion based on HR and baseline wander was defined. Suitable segments for the automatic analysis required that: (i) the difference between the maximum and minimum instantaneous HR in the segment is less than 20 beats/min and (ii) at least $80 \%$ of the beats fulfilled two conditions: the difference between the $i^{t h}$ and the $(i-1)^{t h} R R$ intervals is less than $150 \mathrm{~ms}$ and with a difference between the baseline voltage measured at the PQ segment in that beat and the one measured in the preceding beat lower than $300 \mu \mathrm{V}$.

\subsubsection{TWA waveform estimation}

The method to estimate the TWA waveform is based on [12]. The 8 independent standard leads (V1 to V6, I and II) were linearly combined using periodic component analysis $(\pi \mathrm{CA})$, which maximizes the 2-beat periodicity of the ECG signal (the TWA periodicity) in the combined lead [13]. Then, the Laplacian Likelihood Ratio Metod (LLRM) [14] was applied in the new combined lead to estimate the TWA waveform $\boldsymbol{y}_{k}$ of each segment, denoted as

$$
\boldsymbol{y}_{k}=\left[y_{k}(1), \ldots, y_{k}(N)\right]^{\mathrm{T}},
$$

with $N$ the total number of samples within the ST-T complex.

TWA waveforms, $\boldsymbol{y}_{k}$, may not have the same polarity at each segment and, therefore, might cancel out when averaged. Thus, before final averaging, a phase-alignment step is needed. This phase-aligned waveform, denoted as $\boldsymbol{y}_{k}^{a}$, was estimated as:

$$
\boldsymbol{y}_{k}^{a}=\operatorname{sign}\left(\boldsymbol{y}_{d, k}^{\mathrm{T}} \mathbf{w}_{1}\right) \boldsymbol{y}_{k}
$$

where $\boldsymbol{y}_{d, k}$ is a detrended version of $\boldsymbol{y}_{k}$ and $\mathbf{w}_{1}$ is the dominant waveform, obtained by a classical principal component analysis (PCA) (see [15] for more details).

\subsubsection{The index of average alternans}

Finally, the index of average alternans $I A A$ was defined as the mean absolute value of the average of the phasealigned waveforms $\boldsymbol{y}_{k}^{a}$ :

$$
I A A=\frac{1}{N} \sum_{n=1}^{N}\left|\frac{1}{K} \sum_{k=1}^{K} y_{k}^{a}(n)\right| .
$$



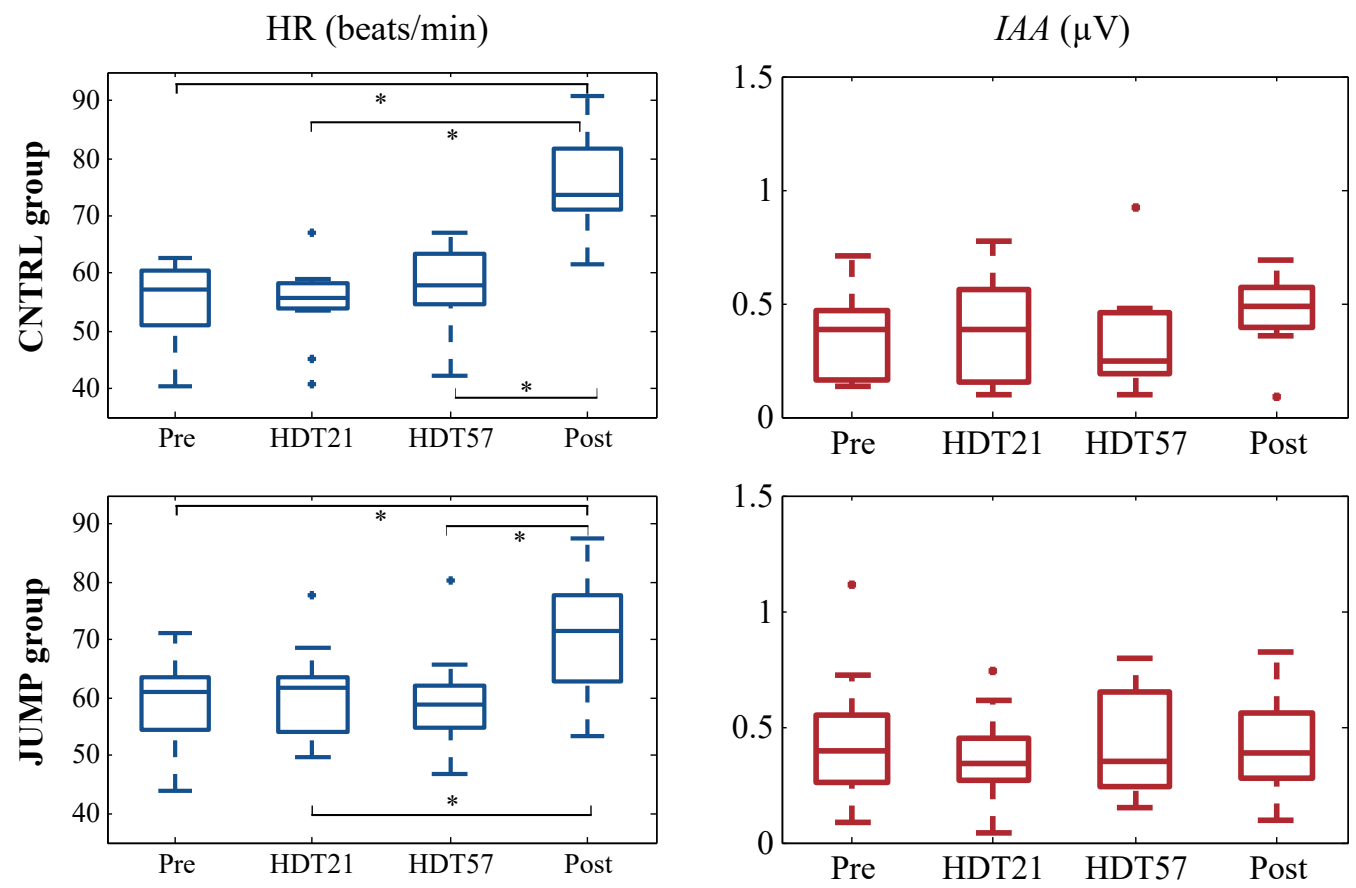

Figure 1. Average HR and IAA values at each time point of the HDBR $(*: p<0.003)$.

\subsection{Statistical analysis}

Data is presented as median $\left(25^{\text {th }} ; 75^{\text {th }}\right.$ percentiles $)$ for continuous variables, unless otherwise specified. Nonparametric Friedman test and Wilcoxon signed rank paired test with Bonferroni correction were applied for repeated measurements to evaluate differences among the different stages of the HDBR (among PRE, HDT21, HDT57 and POST). Mann-Whitney test was applied to evaluate differences between CNTRL and JUMP groups. For all tests, the null hypothesis was rejected for $\mathrm{p} \leq 0.05$.

\section{4. $\quad$ Results}

Average distributions of $\mathrm{HR}$ and $I A A$ computed at each stage of the HDBR are presented on Fig. 1. Results for CNTRL and JUMP groups are presented in upper and bottom panels, respectively.

In CNTRL group, a significant increase in $\mathrm{HR}$ was found at POST compared to PRE $(57.1(50.8 ; 60.6)$ beats/min vs $73.6(70.8 ; 81.6)$ beats/min, $\mathrm{p}=0.003)$, to HDT21 (55.7 (53.7;58.3)beats/min, $\mathrm{p}=0.003)$ and to HDT57 (57.7 $(54.7 ; 63.3)$ beats/min, $\mathrm{p}=0.003)$. A similar pattern was observed with TWA indices. The $I A A$ showed an increasing trend at POST $(0.467(0.381 ; 0.574) \mu \mathrm{V})$ compared to PRE $(0.337(0.204 ; 0.437 \mu \mathrm{V}, \mathrm{p}=0.18)$ and the end of the HDBR (HDT57: $0.247(0.188 ; 0.454) \mu \mathrm{V}, \mathrm{p}=0.09)$, hence not significant. Although HR and TWA have a similar pattern, correlation between both parameters remained low
$\left(R^{2}=0.205\right)$.

In the JUMP group, the induced increase in HR was found at POST $(71.6(62.9 ; 77.8)$ beats/min), in comparison to the rest of HDBR stages (PRE: $61.1(54.48 ; 63.53)$ beat $/ \mathrm{min}, \mathrm{p}=0.002$, HDT21: $61.9(54.1 ; 63.6)$ beats/min, $\mathrm{p}=0.004$ and HDT57: $58.7 \quad(54.9 ; 62.2)$ beats/min, $\mathrm{p}=0.002)$. However, the increasing tendency on $I A A$ observed in CNTRL group, was not visible when the countermeasure was applied.

\section{Discussion and conclusion}

In this study, electrical instability in ventricular repolarization induced by sedentary long-duration HDBR was assessed by the long-term averaging of TWA activity during the night hours. The selection of the night period was motivated by the fact that subjects were asked to perform numerous experimental tests during the day period (especially in PRE and POST periods) that could induce confounding effects. An increasing tendency in $I A A$ at POST with respect to both PRE and to the end of the 60-day immobilization period (HDT57) was observed in the CNTRL group, although it did not reach statistical significance. This increase in TWA could have been induced by the increase observable also on average HR. However, a low correlation between both parameters was measured. Nevertheless, previous reports on which ventricular repolarization heterogeneity was assessed using different repolarization indices (QTc, spatial QRS-T angle, ventricular gradient, 
among others) has been shown to be reversibly altered after short and long-duration HDBR studies [7,8]. These observations might be an indication for initial electrical instability on the myocardial substrate induced by long-term HDBR.

All these changes were less evident when evaluated in the JUMP group, in which subjects were imposed to exercise during 5-6 sessions per week (jump training in a supine position). Already in [10], including the same study population, resting heart rate response to HDBR has been shown to differ between both groups. While in CNTRL group heart rate slowly increased from the middle of the HDBR, with a marked increase at the beginning of the recovery period, in the JUMP group there was no increase or even a decrease in heart rate during the immobilization period, with a slight increase at the beginning of the recovery phase. It is known that an elevated heart rate during or after HDBR was associated with decreased stroke volume, an overall decrease in maximal cardiac output and a decreased cardiac vagal tone with increased sympathetic stimulation and greater cardiac beta-receptor sensitivity [1], factors that also influenced TWA. All of these parameters, on the contrary, can also be influenced by exercise, suggesting that the applied countermeasure could have had a positive impact in the training group, preventing the cardiac deconditioning effects observed in the CNTRL subjects.

To conclude, the absence of significant changes between PRE and POST-HDBR in TWA indices suggests that a 60-day exposure to simulated microgravity is not enough to induce significant changes in healthy myocardial substrate up to the point of reflecting electrical instability in terms of TWA on the surface ECG. Nevertheless, the increasing tendency observed after 60 days of immobilization might be indicative of initial alterations in the repolarization phase. From the clinical perspective, reduced gravitational stimulus during HDBR experiments could also provide interesting information which could be taken into account in immobilized or bedridden patients.

\section{Acknowledgements}

This work was supported by the Italian Space Agency (contract 2013-033-R.0 QT-bed, recipient E.G.Caiani). Also, by CIBER in Bioengineering, Biomaterials \& Nanomedicne (CIBER-BBN) through Instituto de Salud Carlos III and FEDER (Spain), project DPI2016-75458-R funded by MINECO and FEDER, and by Gobierno de Aragón and European Social Fund (EU) through BSICoS Group (T96). The computation was performed by the ICTS NANBIOSIS, specifically by the High Performance Computing Unit of the CIBER-BBN at the University of Zaragoza.

\section{References}

[1] Convertino, V and Hoffler, GW. Cardiovascular physiology. Effects of microgravity. J Fla Med Assoc 1992;79(8):517-524.

[2] Hawkins WR, Zieglschmid JF. Clinical aspects in crew health. Biomedical results of Apollo 1975; (NASA SP-386) 286-304

[3] Nicogossian, AE et al. Space Physiology and Medicine: From Evidence to Practice. Springer-Verlag New York, 2016. 4th Edition.

[4] D'Aunno, D et al. Effect of short- and long-duration spaceflight on QTc intervals in healthy astronauts. Am J Cardiol 2003;91(4):494+

[5] Mitchell, BM and Meck, JV. Short-duration spaceflight does not prolong QTc intervals in male astronauts. Am J Cardiol 2004;93(8):1051-2

[6] Pavy-Le Traon, A et al. From space to Earth: advances in human physiology from 20 years of bed rest studies (1986-2006). Eur J Appl Physiol 2007;101(2):143-194.

[7] Caiani, EG et al. Impaired T-wave amplitude adaptation to heartrate induced by cardiac deconditioning after 5-days of head-down bed-rest. Acta astronautica 2013;91:166-172.

[8] Sakowski, C et al. Sedentary long-duration head-down bed rest and ECG repolarization heterogeneity. Aviat Space Envir Md 2011;82:416-423

[9] Verrier, RL et al. Microvolt T-wave alternans physiological basis, methods of measurement, and clinical utility - Consensus guideline by International Society for Holter and Noninvasive Electrocardiology. J Am Coll Cardiol 2011; 58(13):1309-24

[10] Kramer, A et al. High-intensity jump training is tolerated during 60 Days of bed rest and is very effective in preserving leg power and lean body mass: An overview of the Cologne RSL study. PLOS ONE 12(1): e0169793

[11] Martínez, JP et al. Wavelet-based ECG delineator: evaluation on standard databases. IEEE T Biomed Eng 2004;51:570-581

[12] Monasterio, $\mathrm{V}$ et al. Average T-wave alternans activity in ambulatory ECG records predicts sudden cardiac death in patients with chronic heart failure. Heart rhythm 2012;9(3):383-389.

[13] Monasterio, $\mathrm{V}$ et al. A multilead scheme based on periodic component analysis for T-wave alternans analysis in the ECG. Ann Biomed Eng 2010;38(8):2532-2541

[14] Martínez JP, Olmos S. Methodological principles of T wave alternans analysis: a unified framework. IEEE T Biomed Eng 2005;52(4):599-613

[15] Martín-Yebra, A et al. Post-Ventricular Premature Contraction Phase Correction Improves the Predictive Value of Average T-wave Alternans in Ambulatory ECG Recordings. IEEE T Biomed Eng 2017; DOI:10.1109/TBME.2017.2711645

Address for correspondence:

Alba Martín

DEIB, Politecnico di Milano Piazza Leonardo da Vinci, 32,

20.133, Milano, Italy

albapilar.martin@polimi.it 